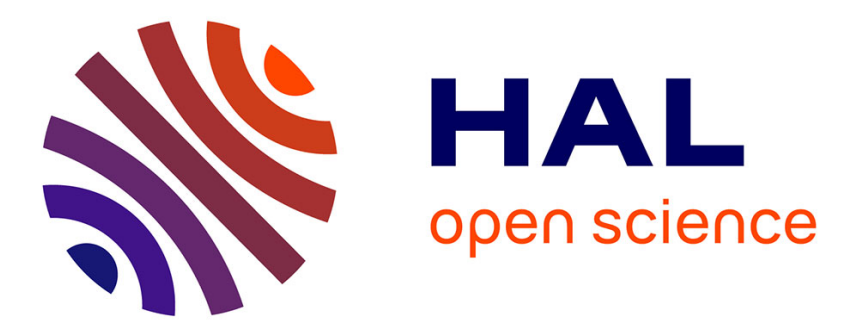

\title{
Informational representability: Abstract models versus concrete models.
}

Stéphane Demri, Ewa Orlowska

\section{To cite this version:}

Stéphane Demri, Ewa Orlowska. Informational representability: Abstract models versus concrete models.. Didier Dubois; Henri Prade; Erich Peter Klement. Fuzzy sets, Logics and Reasoning about Knowledge, 15, Springer, pp.301-314, 1999, Applied Logic Series, 978-94-017-1652-9. 10.1007/97894-017-1652-9_20. hal-03201138

\section{HAL Id: hal-03201138 https://hal.science/hal-03201138}

Submitted on 17 Apr 2021

HAL is a multi-disciplinary open access archive for the deposit and dissemination of scientific research documents, whether they are published or not. The documents may come from teaching and research institutions in France or abroad, or from public or private research centers.
L'archive ouverte pluridisciplinaire HAL, est destinée au dépôt et à la diffusion de documents scientifiques de niveau recherche, publiés ou non, émanant des établissements d'enseignement et de recherche français ou étrangers, des laboratoires publics ou privés. 


\title{
INFORMATIONAL REPRESENTABILITY: ABSTRACT MODELS VERSUS CONCRETE MODELS
}

\author{
Stéphane Demri ${ }^{+} \quad$ Ewa Orłowska** \\ * Institute of Telecommunications \\ Szachowa 1, 04-894 Warsaw, Poland \\ e-mail: orlowska@itl.waw.pl \\ ${ }^{+}$Laboratoire LEIBNIZ - C.N.R.S. \\ 46 Avenue Félix Viallet, 38031 Grenoble Cedex, France \\ e-mail: demri@imag.fr
}

\section{Introduction}

Information logics are modal formalisms for representation of and reasoning about concepts derived from data that describe an application domain. Traditionally, concepts are determined by defining their extension or denotation and intension or connotation. The extension of a concept consists of the objects that are instances of this concept and the intension of a concept consists of the properties that are characteristic for the objects to which this concept applies. For example, to define the concept 'organism' we should list the earmarks of organism and the typical species of organisms [Bun67]. Let a set $O B$ of objects be given, and suppose that properties of those objects are articulated in terms of attributes from a set $A T$ and values of these attributes. For example, property of 'being green' is represented as a pair (colour, green), where 'colour' is an attribute, and 'green' is one of its values. Nondeterministic information about an object is of the form (attribute, a subset of values). For instance, if the age of a person is known approximately, say between 20 and 25, then this information is represented as a pair (age, $\{20, \ldots, 25\})$. By an information system $S$ we understand a pair $(O B, A T)$ where $O B$ is a non-empty set of objects and $A T$ is a non-empty set of attributes. Each attribute $a$ is a mapping $a: O B \rightarrow \mathcal{P}\left(V a l_{a}\right) \backslash\{\emptyset\}$. For each $a \in A T$, the non-empty set $V a l_{a}$ is the set of values of the attribute $a$ [Paw83, OP84]. We write $\mathcal{I S}$ to denote the class of information systems. An information system $S^{\prime}=\left(O B^{\prime}, A T^{\prime}\right)$ is said to be a subsystem of the information system $S=(O B, A T)$ iff $O B^{\prime} \subseteq O B$ and $\left\{a_{O B^{\prime}}: a \in A T\right\}=A T^{\prime}$ where $a_{O B^{\prime}}$ denotes the restriction of $a$ to $O B^{\prime}$.

In various application areas acquisition of concepts is carried on in the situation when the complete information about objects that are supposed to be their instances is not available. There are two major types of incompleteness of information which is given in the form of an information system: indiscernibility and orthogonality. The indiscernibility paradigm emerged from the observation that characterization of objects in terms of their properties might result in indistinguishability: some objects are 'the same' as far as the

\footnotetext{
*The mailing address: Azaliowa 29, 04-539 Warsaw, Poland
} 
admitted properties are concerned. Hence, instead of crisp entities we rather grasp classes of objects such that each class contains those objects that cannot be distinguished one from another in terms of the given properties. It also follows that membership of an object in a set can only be defined modulo the properties of objects, and we might not be able to determine a sharp boundary between a set and its complement. As a consequence we might not be able to assert membership of objects in the extensions of concepts in a two-valued (yes or no) manner. The objects from the classes that are included in a boundary region of the concept can be classified neither as its positive nor negative instances. It follows that we need at least three valued means of reasoning in the presence of indiscernibilitytype incompleteness of information. To model types of incompleteness of information of this kind we introduce a family of relations that reflect impossibility of discerning all the individual objects. The information relations from this group model degrees of indistinguishability in a nonnumerical way and they enable us to define a hierarchy of definability classes of sets [Orło88b, Orło89, Paw91].

However, in many situations it might be more suitable to ask not for indistinguishability but for its opposite. To model in a nonnumerical way degrees of distinguishability we consider a family of orthogonality type relations. The concept of orthogonality plays a crucial role in quantum logic and various classes of logical and algebraic systems have been introduced in this connection [CN89, CDCG93, Gol91]. In those systems orthogonality is a semantic counterpart of negation or complement. In this paper we consider modeling of orthogonality that is related to representation of incomplete information [Orło94]. Both the information relations from the indiscernibility group and the orthogonality group are relative to subsets of attributes. In general, both indiscernibility-type and orthogonalitytype incompleteness of information lead to non-numerical many-valuedness of assertions about objects. The truth value of each assertion depends on a subset of properties of objects that are involved in expressing that assertion. Thus we can view the subsets of properties as non-numerical measures of degrees of truth.

Information logics enable us to represent both extensions and intensions of concepts, and moreover, they exhibit a relevant type of incompleteness of information from which the extensions and the intensions are derived. There are two kinds of semantic structures for information logics: general (or abstract) models and standard models. General models are based on Kripke frames that, however, differ from the usual ones in that their accessibility relations are relative to subsets of a set. This set is intuitively interpreted as the set of attributes that are relevant for the objects from the universe of the frame [Orło88a, Bal97]. The relations are assumed to satisfy various properties, for example indiscernibility relations are equivalence relations, (right) orthogonality relations are the relations whose complements are tolerances, etc. Standard models are based on frames derived from information systems. In these frames the accessibility relations are defined explicitly in terms of the attributes from these systems. Hence, the standard frames are the 'concrete' structures derived directly from data that describe an application domain. A broad family of abstract and standard information frames is presented in [Vak89, Vak91b, Vak91a, Vak97, Orło85, Orło95].

The purpose of this paper is to elaborate a formal framework for expressing and proving informational representability of abstract frames. Let a similarity relation $\triangleright$ in a class $C$ of frames be given, for example relation of 'being isomorphic' or 'being modally equivalent'. Intuitively, a frame $\mathcal{K}$ from the class $\mathrm{C}$ is informationally $\triangleright$-representable if there is an information system and a frame $\mathcal{K}^{\prime}$ derived from this system such that $\mathcal{K}^{\prime}$ is in class $\mathrm{C}$ and, moreover, $\mathcal{K}$ is $\triangleright$-related to $\mathcal{K}^{\prime}$. The first theorem of this kind has been 
proved in [Vak87, Vak89]. In [Orło93] it has been observed that a property of informational representability might be meaningful in investigations of nonclassical logics, and a notion of informational representability has been proposed. In the present paper we introduce a general notion of informational representability, we develop a method of proving informational representability and we give examples of informational representability and non-representability of frames.

An extended version of this paper will appear in [Orło98].

\section{Frames with parameterized accessibility relations}

In this section we introduce a general notion of frame that captures all the types of frames considered in connection with information logics. This notion is an extension of the notion of frame used in the theory of modal logics (see e.g. [Kri63, Che80, vB84, HC84, Gol92]). We shall consider frames with several (finitely many) families of accessibility relations of different arities, and moreover each of these families will be indexed with subsets (and not individual elements) of a set, referred to as the set of parameters. Parameters are intended to be abstract counterparts of entities that determine relations. For example, if we are interested in information relations of an information system, then we should take the attributes of the system as the parameters. If we deal with a logic of knowledge, then the parameters are knowledge agents. Instead of ordinary frames of multimodal logics that contain just several relations, we will be dealing with frames with families of relations. Intuitively, each family consists of relations of the same type, that is all the relations in a family satisfy the same conditions e.g. they are equivalence relations, and in general there are several relations in every such family, each of which is determined by a subset of parameters. For example, the family of indiscernibility relations of an information system consists of relations that reflect indiscernibility of objects with respect to any subset of attributes in that information system. Each of these relations is an equivalence relation.

A signature $\Sigma$ is a pair $\left\langle\mathrm{P},\left\langle n_{1}, \ldots, n_{k}\right\rangle\right\rangle$ where $\mathrm{P}$ is a non-empty set of parameters and $\left\langle n_{1}, \ldots, n_{k}\right\rangle$ is a non-empty sequence of natural numbers greater than 2 . Classes of frames indexing the relations by sets of parameters have been intensively studied in the past (see e.g. [Orło88b, HM92, Bal97]). Let $\Sigma=\left\langle\mathrm{P},\left\langle n_{1}, \ldots, n_{k}\right\rangle\right\rangle$ be a signature. By a $\Sigma$-frame we understand a structure $\left(U,\left\{\mathcal{R}_{l}(P): P \subseteq \mathrm{P}, l \in\{1, \ldots, k\}\right\}\right)$ where $U$ is a non-empty set and for all $P \subseteq \mathrm{P}$, for all $l \in\{1, \ldots, k\}, \mathcal{R}_{l}(P)$ is a $n_{l}$-ary relation on $U$.

We write $\mathcal{F}_{\Sigma}$ to denote the class of $\Sigma$-frames. We also write $\equiv\left(\right.$ resp. $\left.\equiv_{m}\right)$ to denote the isomorphism relation (resp. the modal equivalence relation) between two frames. It is clear that $=\subseteq \subseteq \subseteq \equiv_{m}$.

Example 2.1. Let $S=(O B, A T)$ be an information system. Consider the signature $\Sigma_{0}=\langle A T,\langle 2\rangle\rangle$. Two objects $o_{1}$ and $o_{2}$ are said to be indiscernible with respect to $A \subseteq A T$ (in short $\left.o_{1} \operatorname{ind}(A) o_{2}\right)$ iff for all $a \in A, a\left(o_{1}\right)=a\left(o_{2}\right)$. In the $\Sigma_{0}$-frame $(O B,\{\operatorname{ind}(A): A \subseteq$ $A T\}), \operatorname{ind}(A)$ is an equivalence relation on $O B$ for every $A \subseteq A T$ and for any $A, A^{\prime} \subseteq A T$, $\operatorname{ind}\left(A \cup A^{\prime}\right)=\operatorname{ind}(A) \cap \operatorname{ind}\left(A^{\prime}\right)$.

A $\Sigma$-frame $\mathcal{K}^{\prime}=\left(U^{\prime},\left\{\mathcal{R}_{l}^{\prime}(P): P \subseteq \mathrm{P}, l \in\{1, \ldots, k\}\right\}\right)$ is said to be a subframe of the $\Sigma$-frame $\mathcal{K}=\left(U,\left\{\mathcal{R}_{l}(P): P \subseteq \mathrm{P}, l \in\{1, \ldots, k\}\right\}\right)$ iff $U^{\prime} \subseteq U$ and for all $l \in\{1, \ldots, k\}$, $P \subseteq \mathrm{P}, \mathcal{R}_{l}(P) \cap\left(U^{\prime} \times U^{\prime}\right)=\mathcal{R}_{l}^{\prime}(P)$. 


\section{Frames derived from information systems}

In order to derive $\Sigma$-frames from information systems, a first task consists in relating the set of parameters with a given set of attributes. That is why any derivation of frames shall be defined modulo a contribution function.

Definition 3.1. Let $S=(O B, A T) \in \mathcal{I S}$ and $\Sigma$ be a signature $\left\langle\mathrm{P},\left\langle n_{1}, \ldots, n_{k}\right\rangle\right\rangle$. A contribution function for $S$ is a mapping $I: A T \rightarrow \mathcal{P}(\mathrm{P})$ such that $\bigcup_{a \in A T} I(a)=\mathrm{P}$.

For any $P \subseteq \mathrm{P}$, we write $I^{-1}(P)$ to denote the set $\{a \in A T: I(a) \cap P \neq \emptyset\}$. The intended meaning of $I$ is the following: every attribute $a$ contributes to the construction of relations involving some parameters in $I(a)$. Moreover, every parameter $\mathrm{p}$ has at least one attribute that contributes to $\mathrm{p}$.

\subsection{A language for information systems}

The language LS is determined by seven sets which are supposed to be pairwise disjoint: the set of constants CONS $=\{0,1\}$, the non-empty countable set of variables VAR, the non-empty set of unary function symbols FUN, the set of constructors $\mathrm{OR}=\{\cap, \cup,-\}$ (of respective arity $2,2,1$ ), the set of predicate symbols $\mathrm{PRE}=\{=, \subseteq\}$, the set of quantifiers $\{\forall, \exists\}$ and the set of propositional logical operators $\mathrm{OP}=\{\neg, \wedge\}$. Function symbols are intended to represent the attributes and variables will range over the set of objects of an information system. The set $\mathrm{T}$ of terms is the smallest set that satisfies the following conditions: (1) CONS $\subseteq \mathrm{T}$; (2) if $f \in$ FUN and $x \in \operatorname{VAR}$ then $f(x),-f(x) \in \mathrm{T}$ and (3) for all $\oplus \in \mathrm{OR} \backslash\{-\}$ if $t_{1}, t_{2} \in \mathrm{T}$ then $\oplus\left(t_{1}, t_{2}\right) \in \mathrm{T}$. The set $\mathrm{F}_{0}$ of atomic formulae is the set of expressions $\left\{\oplus\left(t_{1}, t_{2}\right): t_{1}, t_{2} \in \mathrm{T}, \oplus \in \mathrm{PRE}\right\}$. The set $\mathrm{F}$ of formulae is the smallest set that satisfies the following conditions: $\mathrm{F}_{0} \subseteq \mathrm{F}$ and if $\mathrm{c}$ is any $n$-ary propositional operator and $F_{1}, \ldots, F_{n} \in \mathrm{F}$ then $\mathrm{c}\left(F_{1}, \ldots, F_{n}\right) \in \mathrm{F}$. An extended formula is an expression of the form $q_{1} f_{1} \ldots q_{n} f_{n} F$ (also written $\mathcal{Q} F$ ) with $F \in \mathrm{F},\left\{q_{1}, \ldots, q_{n}\right\} \subseteq\{\exists, \forall\}$ and $\left\{f_{1}, \ldots, f_{n}\right\} \subseteq$ FUN. The set of extended formulae is written $\mathrm{F}^{e}$. The extended formula $q_{1} f_{1} \ldots q_{n} f_{n} F$ is said to be weak (resp. strong) iff $\left\{q_{1}, \ldots, q_{n}\right\}=\{\exists\}$ (resp. $\left\{q_{1}, \ldots, q_{n}\right\}=\{\forall\}$ ). For any syntactic set $\mathrm{X}$, and for any syntactic object 0 , we write $\mathrm{X}(0)$ to denote the set consisting of those elements of $\mathrm{X}$ that occur in 0 . An extended formula $\mathcal{Q} F$ is said to be well-closed iff for all $f \in \operatorname{FUN}(F), f$ occurs exactly once in $\mathcal{Q}$. We shall adopt the convention $\mathrm{F} \subseteq \mathrm{F}^{e}$ by considering that a formula is an extended formula with an empty string of quantifications.

Let $S=(O B, A T)$ be an information system. A function interpretation in $S$ is a mapping $m:$ FUN $\rightarrow A T$. An object interpretation in $S$ is a mapping $v: \operatorname{VAR} \rightarrow O B$. The interpretation of terms generated by $m$ and $v$ is the mapping $I_{m, v}: \mathrm{T} \rightarrow \mathcal{P}\left(\bigcup_{a \in A T} V a l_{a}\right)$ such that:

- $I_{m, v}(f(x))=m(f)(v(x)), I_{m, v}(-f(x))=\operatorname{Val}_{m(f)} \backslash I_{m, v}(f(x)), I_{m, v}(0)=\emptyset$,

- $I_{m, v}(1)=\bigcup_{a \in A T} V_{a l}, I_{m, v}\left(\oplus\left(t_{1}, t_{2}\right)\right)=\oplus\left(I_{m, v}\left(t_{1}\right), I_{m, v}\left(t_{2}\right)\right)$ when $\oplus \in \mathrm{OR} \backslash\{-\}$. By abusing our notation, as usual $\cap, \cup,-$ denote the Boolean operations on sets.

It follows that terms represent sets of values of attributes. Let $S=(O B, A T)$ be an information system, $m$ be a function interpretation and $v$ be an object interpretation. We say that an extended formula $F$ is satisfied in $S$ under the interpretation $I_{m, v}$ (written $S, I_{m, v} \models F$ ) when the following conditions are satisfied.

- $S, I_{m, v} \models \oplus\left(t_{1}, t_{2}\right)$ iff $\oplus\left(I_{m, v}\left(t_{1}\right), I_{m, v}\left(t_{2}\right)\right)$ when $\oplus \in \operatorname{PRE}$ 
- $S, I_{m, v} \models \neg F$ iff not $S, I_{m, v} \models F, S, I_{m, v} \models F \wedge G$ iff $S, I_{m, v} \models F$ and $S, I_{m, v} \models G$,

- $S, I_{m, v} \models \forall f F$ iff for all $a \in A T, S, I_{m_{a}^{\prime}, v}=F$ where $m_{a}^{\prime}$ is defined as follows: $m_{a}^{\prime}(f)=a$ and for all $f^{\prime} \neq f, m_{a}^{\prime}\left(f^{\prime}\right)=m\left(f^{\prime}\right)$

- $S, I_{m, v}=\exists f F$ iff there exists $a \in A T$ such that $S, I_{m_{a}^{\prime}, v}=F$ where $m_{a}^{\prime}$ is defined as above.

Let $S=(O B, A T)$ be an information system and $F \in \mathrm{F}^{e}$ such that the variables occurring in $F$ are $x_{1}, \ldots, x_{n}$ (in the order of enumeration). For all function interpretations $m$ for $S$, we write $m_{S}(F)$ to denote the set

$$
m_{S}(F)=\left\{\left\langle v\left(x_{1}\right), \ldots, v\left(x_{n}\right)\right\rangle: \exists v: \operatorname{VAR} \rightarrow O B, S, I_{m, v}=F\right\}
$$

If $F$ has the form $\forall f G$ (resp. $\exists f G$ ) then $m_{S}(F)=\bigcap_{a \in A T}\left(m_{a}^{\prime}\right)_{S}(G)$ (resp. $m_{S}(F)=$ $\left.\bigcup_{a \in A T}\left(m_{a}^{\prime}\right)_{S}(G)\right)$.

ExAmple 3.1. (follows Example 2.1) Consider the formula $f\left(x_{1}\right)=f\left(x_{2}\right)$, say $F_{0}$. Let $S=(O B, A T) \in \mathcal{I S}$. It is easy to show that for all $A \subseteq A T, o_{1}, o_{2} \in O B, o_{1} \operatorname{ind}(A) o_{2}$ iff for all $a \in A,\left(o_{1}, o_{2}\right) \in m_{(O B,\{a\})}\left(F_{0}\right)$ where $m$ is the unique function interpretation in $(O B,\{a\})$.

\section{$3.2 \quad \Sigma$-specification}

The language LS enables us to express definitions of relations derived from information systems. These definitions will be referred to as specifications. Let $\Sigma$ be the signature $\left\langle\mathrm{P},\left\langle n_{1}, \ldots, n_{k}\right\rangle\right\rangle$. A $\Sigma$-specification $\mathrm{S}$ is a sequence of $k$ well-closed extended formulas, say $\left\langle F_{1}, \ldots, F_{k}\right\rangle$ such that for all $l \in\{1, \ldots, k\}, \operatorname{card}\left(\operatorname{VAR}\left(F_{l}\right)\right)=n_{l}$. A $\Sigma$-specification $\mathrm{S}$ is said to be strong (resp. weak) iff $\mathrm{S}$ is a sequence of strong extended formulae (resp. $\mathrm{S}$ is a sequence of either strong or weak extended formulae). Since every extended formula occurring in a $\Sigma$-specification $\left\langle\mathcal{Q}_{1} F_{1}, \ldots, \mathcal{Q}_{k} F_{k}\right\rangle$ is closed with respect to the function symbols, for all $l \in\{1, \ldots, k\}$, for all information systems $S=(O B, A T)$ and for all function interpretations $m, m^{\prime}$ in $S, m_{S}\left(\mathcal{Q}_{l} F_{l}\right)=m_{S}^{\prime}\left(\mathcal{Q}_{l} F_{l}\right)$.

Observe also that when $\operatorname{card}(\operatorname{FUN}(F))=1$, for all $P, P^{\prime} \in \mathcal{P}(\mathrm{P}) \backslash\{\emptyset\}$, if $q_{1} f_{1} F$ is strong (resp. weak) then $m_{\left(O B, I^{-1}\left(P \cup P^{\prime}\right)\right)}\left(q_{1} f_{1} F\right)=\bigcap_{Q \in\left\{P, P^{\prime}\right\}} m_{\left(O B, I^{-1}(Q)\right)}\left(q_{1} f_{1} F\right)$ (resp. $\left.m_{\left(O B, I^{-1}\left(P \cup P^{\prime}\right)\right)}\left(q_{1} f_{1} F\right)=\bigcup_{Q \in\left\{P, P^{\prime}\right\}} m_{\left(O B, I^{-1}(Q)\right)}\left(q_{1} f_{1} F\right)\right)$. Moreover, for any information system $S=(O B, A T)$ and for any contribution function $I$ for $S$, if $\operatorname{card}(A T)=1$ then for all extended formulae $\mathcal{Q}_{1} F$ and $\mathcal{Q}_{2} F$ and for all $\emptyset \neq P \subseteq \mathrm{P}, m_{\left(O B, I^{-1}(P)\right)}\left(\mathcal{Q}_{1} F\right)=$ $m_{\left(O B, I^{-1}(P)\right)}\left(\mathcal{Q}_{2} F\right)=m_{\left(O B, I^{-1}(P)\right)}(F)$. In the sequel, we write $\operatorname{spec}_{\Sigma}^{1}$ to denote the set of $\Sigma$-specifications $\left\langle F_{1}, \ldots, F_{k}\right\rangle$ such that for all $l \in\{1, \ldots, k\}, \operatorname{card}\left(\operatorname{FUN}\left(F_{l}\right)\right)=1$.

\subsection{A frame derived from an information system}

We are now in position to define a family of derivation functions parametrized by signatures and specifications.

Definition 3.2. Let $\Sigma=\left\langle\mathrm{P},\left\langle n_{1}, \ldots, n_{k}\right\rangle\right\rangle$ be a signature, $S=(O B, A T) \in \mathcal{I} \mathcal{S}, I$ be a contribution function for $S$ and $\mathrm{S}=\left\langle F_{1}, \ldots, F_{k}\right\rangle$ be a $\Sigma$-specification. We write $D_{\Sigma, \mathrm{S}}(S, I)$ to denote the $\Sigma$-frame $\left(O B,\left\{\mathcal{R}_{l}(P): P \subseteq \mathrm{P}, l \in\{1, \ldots, k\}\right\}\right)$ such that

$$
\forall l \in\{1, \ldots k\}, \forall \emptyset \neq P \subseteq \mathrm{P}, \mathcal{R}_{l}(P)=m_{\left(O B, I^{-1}(P)\right)}\left(F_{l}\right)
$$


By convention, for all $l \in\{1, \ldots k\}$, if $F_{l}$ is weak then $\mathcal{R}_{l}(\emptyset)=\emptyset$ otherwise $\mathcal{R}_{l}(\emptyset)=$ $O B \times O B$

It is important to remember that the correctness of Definition 3.2 rests on the fact that each $F_{l}$ is closed with respect to the function symbols.

\section{Informational representability of $\Sigma$-frames}

In this section we present a notion of informational representability of a class of frames and a general method of proving representability. Next, the method will be applied to some particular classes of frames.

Definition 4.1. Let $\Sigma$ be a signature, $X \subseteq \mathcal{F}_{\Sigma}, Y \subseteq \mathcal{I S}$, and $\mathrm{S}=\left\langle F_{1}, \ldots, F_{k}\right\rangle$ be a $\Sigma$-specification. The class of $\Sigma$-frames $X$ is said to be $(\triangleright, \mathrm{S})$-inf-representable $(\triangleright \in\{=, \equiv$ ,$\left.\left.\equiv_{m}\right\}\right)$ in $Y$ iff

1. (soundness) for all $S \in Y$ and for all contribution functions $I$ for $S, D_{\Sigma, \mathrm{S}}(S, I) \in X$,

2. (completeness) for all $\mathcal{K} \in X$, there is $S \in Y$ and a contribution function $I$ for $S$ such that $D_{\Sigma, \mathrm{S}}(S, I) \triangleright \mathcal{K}$.

\subsection{Nice pair proof technique}

This technique has been originally introduced in [Vak87] for the logic NIL. For all nonempty sets $X$, for all $\langle\mathrm{p}, Y\rangle \in \mathrm{P} \times \mathcal{P}(\mathcal{P}(X))$, we write at $t^{\langle\mathrm{p}, Y\rangle}$ to denote the mapping $X \rightarrow \mathcal{P}(\{\mathrm{p}\} \times Y)$ such that for all $x \in X, a t{ }^{\langle\mathrm{p}, Y\rangle}(x)=\{\langle\mathrm{p}, Z\rangle: x \in Z \in Y\}$. Hence for all $Z \in Y, x \in X, x \in Z$ iff $\langle\mathrm{p}, Z\rangle \in a t^{\langle\mathrm{p}, Y\rangle}(x)$.

Definition 4.2. Let $\mathcal{K}=\left(U,\left\{\mathcal{R}_{l}(P): P \subseteq \mathrm{P}, l \in\{1, \ldots, k\}\right\}\right) \in \mathcal{F}_{\Sigma}$ and $\mathrm{S}=\left\langle F_{1}, \ldots, F_{k}\right\rangle \in$ $\operatorname{spec}_{\Sigma}^{1}$. A nice pair with respect to $\mathcal{K}$ and $\mathrm{S}$, say $\mathcal{N}=\langle\mathrm{p}, X\rangle$, is a member of $\mathrm{P} \times \mathcal{P}(\mathcal{P}(U))$

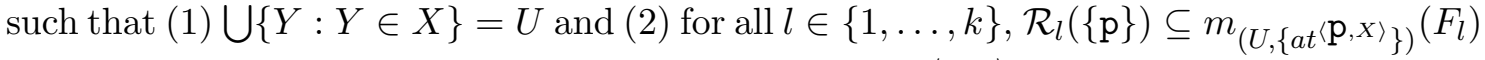
where $m$ is the unique function interpretation in $\left(U,\left\{a t^{\langle\mathbf{p}, X\rangle}\right\}\right)$.

Definition 4.3 below presents different kinds of nice pairs and sets of nice pairs.

Definition 4.3. With the notations of Definition 4.2, a nice pair $\mathcal{N}$ is $(\mathcal{K}, \mathrm{S})$-complete with respect to the parameter $\mathrm{p}$ iff the inclusion in Definition $4.2(2)$ is replaced by an equality. A set of nice pairs $X$ is said to be $(\mathcal{K}, \mathrm{S})$-complete iff for all $\mathrm{p} \in \mathrm{P}$, there is $(\mathrm{p}, Y) \in X$ such that $(\mathrm{p}, Y)$ is $(\mathcal{K}, \mathrm{S})$-complete with respect to $\mathrm{p}$. A set of nice pairs $X$ is said to be $\mathrm{P}$-full iff for all $\mathrm{p} \in \mathrm{P},\left\{\left\langle\mathrm{p}^{\prime}, Y\right\rangle \in X: \mathrm{p}=\mathrm{p}^{\prime}\right\} \neq \emptyset$. A set of nice pairs $X$ is minimally $(\mathcal{K}, \mathrm{S})$-complete iff $X$ is $(\mathcal{K}, \mathrm{S})$-complete and for all $Y \subset X, Y$ is not $(\mathcal{K}, \mathrm{S})$-complete.

Definition 4.4 shall be mainly needed in Section 4.2 .

Definition 4.4. Let $\Sigma$ be a signature, $\mathrm{S}=\left\langle F_{1}, \ldots, F_{k}\right\rangle \in \operatorname{spec}_{\Sigma}^{1}$ and $X \subseteq \mathcal{F}_{\Sigma}$. A (resp. minimal) nice pair function with respect to $\mathrm{S}$ and $X$ is a mapping truc such that for all $\mathcal{K} \in X, \operatorname{truc}(\mathcal{K})$ is a (resp. minimally) $(\mathcal{K}, \mathrm{S})$-complete set of nice pairs 
ExAmPle 4.1. (Example 3.1 continued) Consider the $\Sigma_{0}$-specification $\mathrm{S}_{0}=\left\langle\forall f F_{0}\right\rangle$ with $\Sigma_{0}=\langle\mathrm{P},\langle 2\rangle\rangle$ for some set of parameters $\mathrm{P}=\{\mathrm{p}\}$. Let $\mathcal{K}=\left(U,\left\{\mathcal{R}_{l}(P): P \subseteq\{\mathrm{p}\}, l \in\right.\right.$ $\{1\}\})$ be a $\Sigma_{0}$-frame such that $\mathcal{R}_{1}(\emptyset)=U \times U$ and $\mathcal{R}_{1}(\{\mathrm{p}\})$ is an equivalence relation. Consider the pair $\left\langle\mathrm{p},\left\{\mathcal{R}_{1}(\{\mathrm{p}\})(u): u \in U\right\}\right\rangle=\langle\mathrm{p}, X\rangle$. Since $\mathcal{R}_{1}(\{\mathrm{p}\})$ is reflexive, then $\bigcup_{Y \in X} Y=U$. Moreover, for all $u, v \in U$

$$
\begin{aligned}
(u, v) \in \mathcal{R}_{1}(\{\mathrm{p}\}) & \text { iff } \mathcal{R}_{1}(\{\mathrm{p}\})(u)=\mathcal{R}_{1}(\{\mathrm{p}\})(v) \\
& \text { iff }\{Y: u \in Y \in X\}=\{Y: v \in Y \in X\} \\
& \text { iff }\{\langle\mathrm{p}, Y\rangle: u \in Y \in X\}=\{\langle\mathrm{p}, Y\rangle: v \in Y \in X\} \\
& \text { iff } a t^{\mathrm{p}, X\rangle}(u)=a t^{\langle\mathrm{p}, X\rangle}(v) \\
& \text { iff } \left.(u, v) \in m_{(U,\{a t}\left\langle^{\mathrm{p}, X\rangle}\right\}\right)
\end{aligned}
$$

So $\langle\mathrm{p}, X\rangle$ is $\left(\mathcal{K}, \mathrm{S}_{0}\right)$-complete with respect to $\mathrm{p}$ and $\{\langle\mathrm{p}, X\rangle\}$ is a minimally $\left(\mathcal{K}, \mathrm{S}_{0}\right)$-complete set of nice pairs.

Proposition 4.1. With the notations of Definition 4.2, let $X$ be a P-full set of nice pairs. Then, (1) $S_{X}=\left(U,\left\{a t^{\langle\mathrm{p}, Y\rangle}:\langle\mathrm{p}, Y\rangle \in X\right\}\right)$ is an information system and (2) $I_{X}:\left\{a t^{\langle\mathrm{p}, Y\rangle}:\langle\mathrm{p}, Y\rangle \in X\right\} \rightarrow \mathcal{P}(\mathrm{P})$ with $I_{X}\left(a t^{\langle\mathrm{p}, Y\rangle}\right)=\{\mathrm{p}\}$, is a contribution function for $S_{X}$.

Observe that for any $\langle\mathrm{p}, Y\rangle,\left\langle\mathrm{p}^{\prime}, Y^{\prime}\right\rangle \in X$ with $\mathrm{p} \neq \mathrm{p}^{\prime}$, at ${ }^{\langle\mathrm{p}, Y\rangle} \neq a t^{\left\langle\mathrm{p}^{\prime}, Y^{\prime}\right\rangle} . S_{X}$ is a set-theoretical information system following Vakarelov's terminology (see e.g. [Vak95, Vak97]). Lemma 4.2 below can be seen as the main technical result of the paper since it establishes correspondences between $\Sigma$-frames and set-theoretical information systems obtained from complete set of nice pairs (using the language LS). Proposition 4.3 states some consequences for the informational representability.

Lemma 4.2. Let $\Sigma$ be a signature, $\mathcal{K}=\left(U,\left\{\mathcal{R}_{l}(P): P \subseteq \mathrm{P}, l \in\{1, \ldots, k\}\right\}\right) \in \mathcal{F}_{\Sigma}$, $\mathrm{S}=\left\langle F_{1}, \ldots, F_{k}\right\rangle$ be a weak $\Sigma$-specification in $\operatorname{spec}_{\Sigma}^{1}$ and $X$ be a set of nice pairs with respect to $\mathcal{K}$ and S. Moreover, assume that for all $l \in\{1, \ldots, k\}$, for all $\emptyset \neq P \subseteq \mathrm{P}$, if $F_{l}$ is strong then $\mathcal{R}_{l}(P)=\bigcap_{\mathrm{p} \in P} \mathcal{R}_{l}(\{\mathrm{p}\})$ otherwise $\mathcal{R}_{l}(P)=\bigcup_{\mathrm{p} \in P} \mathcal{R}_{l}(\{\mathrm{p}\})$.

1. If $\mathrm{S}$ is strong and $X$ is $(\mathcal{K}, \mathrm{S})$-complete then

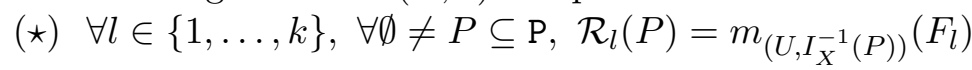

2. If $X$ is minimally $(\mathcal{K}, \mathrm{S})$-complete then $(\star)$.

Proposition 4.3 below states sufficient conditions to establish informational representability.

Proposition 4.3. Let $\Sigma$ be a signature, $\mathrm{S}=\left\langle F_{1}, \ldots, F_{k}\right\rangle$ be a strong (resp. weak) $\Sigma$ specification in $\operatorname{spec}_{\Sigma}^{1}$. Let $X \subseteq \mathcal{F}_{\Sigma}$ such that for all $l \in\{1, \ldots, k\}$, for all $\emptyset \neq P \subseteq \mathrm{P}$, for all $\mathcal{K}=\left(U,\left\{\mathcal{R}_{l}(P): P \subseteq \mathrm{P}, l \in\{1, \ldots, k\}\right\}\right) \in X$, if $F_{l}$ is strong then $\mathcal{R}_{l}(P)=\bigcap_{\mathrm{p} \in P} \mathcal{R}_{l}(\{\mathrm{p}\})$ and $\mathcal{R}_{l}(\emptyset)=U \times U$ otherwise $\mathcal{R}_{l}(P)=\bigcup_{\mathrm{p} \in P} \mathcal{R}_{l}(\{\mathrm{p}\})$ and $\mathcal{R}_{l}(\emptyset)=\emptyset$. Let $Y \subseteq \mathcal{I} \mathcal{S}$ and $\triangleright \in\left\{=, \equiv, \equiv_{m}\right\}$ such that:

1. For all $S \in Y$, for all contribution functions $I$ for $S$, we have $D_{\Sigma, \mathrm{S}}(S, I) \in X$.

2. there is a (resp. minimal) nice pair function with respect to $\mathrm{S}$ and $X$, say truc, such that $\left\{S_{\operatorname{truc}(\mathcal{K})}: \exists \mathcal{K} \in X\right\} \subseteq Y . S_{\operatorname{truc}(\mathcal{K})}$ is defined as in Proposition 4.1.

Then $X$ is $(\triangleright, \mathrm{S})$-inf-representable in $Y$. 
ExAmple 4.2. (Example 4.1 continued) We write $X_{0}$ to denote the set of $\Sigma_{0}$-frames such that $\mathcal{R}_{1}(\emptyset)=U \times U$ and $\mathcal{R}_{1}(\{\mathrm{p}\})$ is an equivalence relation. We show that $X_{0}$ is $\left(=, \mathrm{S}_{0}\right)$-inf-representable in $\mathcal{I S}$. For any information system $S$, and for any contribution function $I$ (actually there is a only one), it is easy to check that $D_{\Sigma_{0}, \mathrm{~S}_{0}}(S, I) \in X_{0}$. For each $\mathcal{K} \in X_{0}$, we build the pair $\operatorname{truc}(\mathcal{K})=\langle\mathrm{p}, X\rangle$ as in Example 4.1 From Example 4.1, for each $\mathcal{K} \in X_{0},\{\operatorname{truc}(\mathcal{K})\}$ is a minimally $\left(\mathcal{K}, \mathrm{S}_{0}\right)$-complete set of nice pairs. $\left\{S_{\text {truc }(\mathcal{K})}\right.$ : $\mathcal{K} \in X\} \subseteq \mathcal{I S}$ by Proposition 4.1 and by Proposition $4.3, X_{0}$ is $\left(=, \mathrm{S}_{0}\right)$-inf-representable in $\mathcal{I S}$.

\subsection{Negation and reordering lemmas}

In this section, we present two ways to obtain (minimal) nice pair functions from existing ones by relating adequately the specifications and classes of frames. In that way, we facilitate the application of Proposition 4.3.

Let $F \in \mathrm{F}^{e}$ such that $\operatorname{VAR}(F)=\left\{x_{1}, \ldots, x_{n}\right\}, x_{1}, \ldots, x_{n}$ being in the order of enumeration. For any permutation $\sigma$ of set $\{1, \ldots, n\}$ we write $F \sigma$ to denote the formula obtained from $F$ by substituting simultaneously in $F, x_{i}$ by $x_{\sigma(i)}$ for all $i \in\{1, \ldots, n\}$. Moreover, for any $n$-ary relation $R$ and for any permutation $\sigma$ of set $\{1, \ldots, n\}$ we write $R \sigma$ to denote the following $n$-ary relation:

$$
R \sigma=\left\{\left(o_{\sigma(1)}, \ldots, o_{\sigma(n)}\right):\left(o_{1}, \ldots, o_{n}\right) \in R\right\}
$$

Observe that for any information system $S=(O B, A T)$, for any function interpretation $m$ in $S, m_{S}(F) \sigma=m_{S}(F \sigma)$. For any $\Sigma$-specification $\mathrm{S}=\left\langle F_{1}, \ldots, F_{k}\right\rangle$, for any set of permutations $\left\{\sigma_{l}:\left\{1, \ldots, n_{l}\right\} \rightarrow\left\{1, \ldots, n_{l}\right\}, l \in\{1, \ldots, k\}\right\}$, for any $\Sigma$-frame $\mathcal{K}=\left(U,\left\{\mathcal{R}_{l}(P): P \subseteq \mathrm{P}, l \in\{1, \ldots, k\}\right\}\right)$ we write $\mathcal{K} \sigma_{1} \ldots \sigma_{k}=\left(U,\left\{\mathcal{R}_{l}^{\prime}(P): P \subseteq\right.\right.$ $\mathrm{P}, l \in\{1, \ldots, k\}\})$ to denote the $\Sigma$-frame such that for any $l \in\{1, \ldots, k\}$, for any $P \subseteq \mathrm{P}$, $\mathcal{R}_{l}^{\prime}(P)=\mathcal{R}_{l}(P) \sigma_{l}$.

LEMma 4.4. (Reordering) Let $\Sigma$ be a signature, $\mathrm{S}=\left\langle F_{1}, \ldots, F_{k}\right\rangle$ be a weak $\Sigma$-specification in $\operatorname{spec}_{\Sigma}^{1}, X \subseteq \mathcal{F}_{\Sigma}$, and truc be a minimal nice pair function with respect to $\mathrm{S}$ and $X$. Let $\sigma_{l}$ be a permutation of the set $\left\{1, \ldots, n_{l}\right\}$ for all $l \in\{1, \ldots, k\}$. Then truc' is a minimal nice pair function with respect to $\mathrm{S}^{\prime}=\left\langle F_{1} \sigma_{1}, \ldots, F_{k} \sigma_{k}\right\rangle$ and $\left\{\mathcal{K} \sigma_{1} \ldots \sigma_{k}: \mathcal{K} \in X\right\}$ where $\operatorname{truc}^{\prime}\left(\mathcal{K} \sigma_{1} \ldots \sigma_{k}\right)=\operatorname{truc}(\mathcal{K})$ for all $\mathcal{K} \in X$.

The starting point of Lemma 4.5 below rests on the fact that for any $S=(O B, A T) \in$ $\mathcal{I S}$, for any function interpretation in $S, m, m_{S}(\neg F)=O B^{\operatorname{card}(\operatorname{VAR}(F))} \backslash m_{S}(F)$ with $F \in \mathrm{F}$. For any $\Sigma$-specification $\mathrm{S}=\left\langle F_{1}, \ldots, F_{k}\right\rangle$, for any $\Sigma$-frame $\mathcal{K}=\left(U,\left\{\mathcal{R}_{l}(P): P \subseteq\right.\right.$ $\mathrm{P}, l \in\{1, \ldots, k\}\})$ we write $\mathcal{K}\urcorner=\left(U,\left\{\mathcal{R}_{l}^{\prime}(P): P \subseteq \mathrm{P}, l \in\{1, \ldots, k\}\right\}\right)$ to denote the $\Sigma$-frame such that

- if $F_{l}$ is weak then $\mathcal{R}_{l}^{\prime}(\emptyset)=\emptyset$ otherwise $\mathcal{R}_{l}^{\prime}(\emptyset)=U \times U$,

- for all $\emptyset \neq P \subseteq$ P, if $F_{l}$ is strong then $\mathcal{R}_{l}^{\prime}(P)=\bigcap_{\mathrm{p} \in P} \mathcal{R}_{l}^{\prime}(\{\mathrm{p}\})$ otherwise $\mathcal{R}_{l}^{\prime}(P)=$ $\bigcup_{\mathrm{p} \in P} \mathcal{R}_{l}^{\prime}(\{\mathrm{p}\})$,

- for all $\mathrm{p} \in \mathrm{P} ; \mathcal{R}_{l}^{\prime}(\{\mathrm{p}\})=-\mathcal{R}_{l}(\{\mathrm{p}\})$.

Lemma 4.5. (Negation) Let $\Sigma$ be a signature, $\mathrm{S}=\left\langle q_{1} f_{1} F_{1}, \ldots, q_{k} f_{k} F_{k}\right\rangle$ be a weak $\Sigma$-specification in $\operatorname{spec}_{\Sigma}^{1}, X \subseteq \mathcal{F}_{\Sigma}$ and truc be a minimal nice pair function with respect to $\mathrm{S}$ and $X$. Then truc ${ }^{\prime}$ is a minimal nice pair function with respect to $\mathrm{S}^{\prime}=$ $\left\langle q_{1} f_{1} \neg F_{1}, \ldots, q_{k} f_{k} \neg F_{k}\right\rangle$ and $\left.\{\mathcal{K}\urcorner: \exists \mathcal{K} \in X\right\}$ where $\left.\operatorname{truc}^{\prime}(\mathcal{K}\urcorner\right)=\operatorname{truc}(\mathcal{K})$ for all $\mathcal{K} \in X$. 


\section{Examples of informational representability}

We present examples of informational representability theorems for frames with information relations from the indiscernibility group and the orthogonality group. The analogous representability results for many other classes of frames can be obtained using the method developed in the paper.

In the rest of the section, $\Sigma$ is assumed to be a signature $\langle\mathrm{P},\langle 2\rangle\rangle$ for some non-empty set of parameters $\mathrm{P}$, unless otherwise stated. Concerning the indiscernibility relation extensively used in the literature, the reader is invited to check the previous examples.

\subsection{Complementarity}

Let $S=(O B, A T) \in \mathcal{I S}$. Two objects $o_{1}$ and $o_{2}$ are said to be in relation of complementarity with respect to $A \subseteq A T$ (in short $o_{1} \operatorname{comp}(A) o_{2}$ ) iff for all $a \in A, a\left(o_{1}\right)=\operatorname{Val}_{a} \backslash a\left(o_{2}\right)$. With LS, $\operatorname{comp}(A)=m_{(O B, A)}\left(F_{0}\right)$ with $F_{0}=\forall f f\left(x_{1}\right)=-f\left(x_{2}\right)$. Observe that $\operatorname{comp}(A)$ is symmetrical, irreflexive and intransitive. When $o_{1} \operatorname{comp}(A) o_{2}$ holds, for all $a \in A, V_{a l}$ is uniquely determined by $a\left(o_{1}\right)$ and $a\left(o_{2}\right)\left(V a l_{a}\right.$ is then the union of $a\left(o_{1}\right)$ and $\left.a\left(o_{2}\right)\right)$. This may explain why the representation of complementary relations has been an open problem until now [Vak97]. We define a class of abstract $\Sigma$-frames related to the $\Sigma$-specification $\mathrm{S}_{0}=\left\langle F_{0}\right\rangle$. First, some preliminary definitions are needed. Let $R$ be a binary relation over the set $U, R$ is said to be complementary iff $R$ is symmetrical, $R$ does not contain cycles of odd length and for all $u, v, w, z \in U$, if $(u, v) \in R,(v, w) \in R$ and $(w, z) \in R$ then $(u, z) \in R$ (3-transitivity). It can be shown that $\operatorname{comp}(A T)$ is a complementary relation. We write $\mathcal{F}_{\mathrm{S}_{0}}$ to denote the set of $\Sigma$-frames $\left(U,\left\{\mathcal{R}_{l}(P): P \subseteq \mathrm{P}, l \in\{1\}\right\}\right)$ such that, $\mathcal{R}_{1}(\emptyset)=U \times U$, for all $\emptyset \neq P \subseteq \mathrm{P}, \mathcal{R}_{1}(P)=\bigcap_{\mathrm{p} \in P} \mathcal{R}_{1}(\{\mathrm{p}\})$ and for all $\mathrm{p} \in \mathrm{P}, \mathcal{R}_{1}(\{\mathrm{p}\})$ is complementary. The rest of the section is devoted to show that $\mathcal{F}_{\mathrm{S}_{0}}$ is $\left(=, \mathrm{S}_{0}\right)$-inf-representable in $\mathcal{I} \mathcal{S}$. For any binary relation $R$ over the set $U$, for all $u \in U$ we write $C_{u, R}$ to denote the largest subset of $U$ such that $u \in C_{u, R}$ and for all $v \in C_{u, R} \backslash\{u\}$, there is a $R^{\prime}$-path between $u$ and $v$ where $R^{\prime}$ is the symmetrical closure of $R$. Observe that $\left\{C_{u, R}: u \in U\right\}$ is a partition of $U$. For all $u \in U$, we write $C_{u, R}^{0}$ (resp. $C_{u, R}^{1}$ ) to denote the largest subset of $C_{u, R}$ such that for all $v \in C_{u, R}^{0}$, there is a $R^{\prime}$-path of even (resp. odd) length between $u$ and $v$ where $R^{\prime}$ is the symmetrical closure of $R$. Moreover, for all $U^{\prime} \subseteq U$, we write $C_{u, R}^{U^{\prime}}$ to denote $C_{u, R}^{0}$ if $u \in U^{\prime}, C_{u, R}^{1}$ otherwise. Observe that for all $u, v, w \in U$, when $R$ is complementary, if $\{v, w\} \subseteq C_{u, R}$ then $\left\{C_{v, R}^{1}, C_{v, R}^{0}\right\}=\left\{C_{w, R}^{1}, C_{w, R}^{0}\right\}$.

Lemma 5.1. Let $R$ be a complementary relation over the set $U$. The set $\left\{C_{u, R}^{i}: u \in\right.$ $U, i \in\{0,1\}\}$ is a partition of $U$.

Let truc be the mapping such that for all $\mathcal{K}=\left(U,\left\{\mathcal{R}_{l}(P): P \subseteq \mathrm{P}, l \in\{1\}\right\}\right) \in \mathcal{F}_{\mathrm{S}_{0}}$,

$$
\operatorname{truc}(\mathcal{K})=\left\{\left\langle\mathrm{p},\left\{\bigcup_{u \in Y} C_{u, \mathcal{R}_{1}(\{\mathrm{p}\})}^{U^{\prime}}: \exists U^{\prime} \subseteq U, \exists Y \in W_{\mathrm{p}}\right\}\right\rangle: \mathrm{p} \in \mathrm{P}\right\}
$$

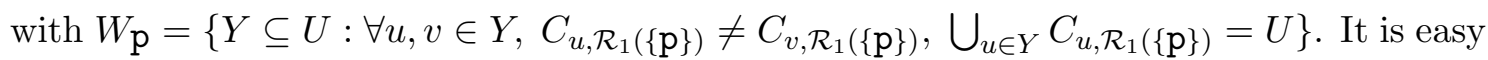
to show that for all $\langle\mathrm{p}, X\rangle \in \operatorname{truc}(\mathcal{K})$, for all $Y_{0} \in W_{\mathrm{p}}, X=\left\{\bigcup_{u \in Y_{0}} C_{u, \mathcal{R}_{1}(\{\mathrm{p}\})}^{U^{\prime}}: \exists U^{\prime} \subseteq U\right\}$. It is worth mentioning that, for all $u, v \in U,(\forall Y \in X$, either $u \in Y$ and $v \notin Y$ or $u \notin Y$ and $v \in Y)$ iff $\left.\left.\left.(u, v) \in m_{(U,\{a t} \mathbf{p}, X\right\rangle\right\}\right)\left(F_{0}\right)$. Moreover, for all $\langle\mathrm{p}, X\rangle \in \operatorname{truc}(\mathcal{K}), \bigcup_{Y \in X} Y=U$. 
Lemma 5.2. Let $\mathcal{K}=\left(U,\left\{\mathcal{R}_{l}(P): P \subseteq \mathrm{P}, l \in\{1\}\right\}\right) \in \mathcal{F}_{\mathrm{S}_{0}}$. For all $\langle\mathrm{p}, X\rangle \in \operatorname{truc}(\mathcal{K})$, for all $u, v \in U,(u, v) \in \mathcal{R}_{1}(\{\mathrm{p}\})$ iff for all $Y \in X$, either $(u \in Y$ and $v \notin Y)$ or $(u \notin Y$ and $v \in Y)$.

Lemma 5.3. Let $\mathcal{K}=\left(U,\left\{\mathcal{R}_{l}(P): P \subseteq \mathrm{P}, l \in\{1\}\right\}\right) \in \mathcal{F}_{\mathrm{S}_{0}} \cdot \operatorname{truc}(\mathcal{K})$ is a minimally $\left(\mathcal{K}, \mathrm{S}_{0}\right)$-complete set of nice pairs.

Corollary 5.4. $\mathcal{F}_{\mathrm{S}_{0}}$ is $\left(=, \mathrm{S}_{0}\right)$-inf-representable in $\mathcal{I S}$.

For any $\mathrm{p} \in \mathrm{P}$ the class of frames $\mathcal{F}_{\mathrm{p}}$ (in the standard sense for modal logics) defined by $\mathcal{F}_{\mathrm{p}}=\left\{\left(U, \mathcal{R}_{1}(\{\mathrm{p}\})\right):\left(U,\left\{\mathcal{R}_{l}(P): P \subseteq \mathrm{P}, l \in\{1\}\right\}\right) \in \mathcal{F}_{\mathrm{S}_{0}}\right\}$ is not closed by the p-morphism construction. It follows that $\mathcal{F}_{\mathrm{p}}$ is not modally definable (see [GT75]). The condition of being a complementary relation can be expressed by a set $\Gamma$ of formulas from the classical first-order logic.

Let $S=(O B, A T) \in \mathcal{I S}$. Two objects $o_{1}$ and $o_{2}$ are said to be in relation of incomplementarity with respect to $A \subseteq A T$ (in short $o_{1}$ incomp $\left.(A) o_{2}\right)$ iff for all $a \in A,\left(V a l_{a} \backslash\right.$ $\left.a\left(o_{1}\right)\right) \neq a\left(o_{2}\right)$. With LS, incomp $(A)=m_{S}\left(F_{0}^{\prime}\right)$ with $F_{0}^{\prime}=\forall f \neg\left(-f\left(x_{1}\right)=f\left(x_{2}\right)\right)$. Using Lemma 4.5 and the above construction, $\left.\{\mathcal{K}\urcorner: \exists \mathcal{K} \in \mathcal{F}_{\mathbf{S}_{0}}\right\}$ is $\left(=,\left\langle F_{0}^{\prime}\right\rangle\right)$-inf-representable in $\mathcal{I S}$.

\subsection{Positive and negative similarity}

Let $S=(O B, A T) \in \mathcal{I S}$. Two objects $o_{1}$ and $o_{2}$ are said to be in relation of positive similarity (resp. negative similarity) with respect to $A \subseteq A T$ (in short $o_{1} p \operatorname{pim}(A) o_{2}$ -resp. $\left.o_{1} n \operatorname{sim}(A) o_{2}\right)$ iff for all $a \in A, a\left(o_{1}\right) \cap a\left(o_{2}\right) \neq \emptyset\left(\operatorname{resp} .\left(V_{a l} \backslash a\left(o_{1}\right)\right) \cap\left(\operatorname{Val}_{a} \backslash\right.\right.$ $\left.\left.a\left(o_{2}\right)\right) \neq \emptyset\right)$. With LS, $\operatorname{psim}(A)=m_{(O B, A)}\left(F_{0}\right)\left(\right.$ resp. $\left.n \operatorname{sim}(A)=m_{(O B, A)}\left(F_{0}\right)\right)$ with $F_{0}=\forall f f\left(x_{1}\right) \cap f\left(x_{2}\right) \neq 0$ (resp. $F_{0}=\forall f-f\left(x_{1}\right) \cap-f\left(x_{2}\right) \neq 0$ ). Let truc be the mapping such that for all $\mathcal{K}=\left(U,\left\{\mathcal{R}_{l}(P): P \subseteq \mathrm{P}, l \in\{1\}\right\}\right) \in \mathcal{F}_{\Sigma}$,

$$
\begin{gathered}
\operatorname{truc}(\mathcal{K})=\left\{\left\langle\mathrm{p},\left\{\{u, v\}:(u, v) \in \mathcal{R}_{1}(\{\mathrm{p}\})\right\}\right\rangle: \mathrm{p} \in \mathrm{P}\right\} \\
\left(\text { resp. } \operatorname{truc}(\mathcal{K})=\left\{\left\langle\mathrm{p},\left\{U \backslash\{u, v\}:(u, v) \in \mathcal{R}_{1}(\{\mathrm{p}\})\right\} \cup\{U\}\right\rangle: \mathrm{p} \in \mathrm{P}\right\}\right)
\end{gathered}
$$

We write $\mathcal{F}_{\mathrm{S}_{0}}$ to denote the set of $\Sigma$-frames $\left(U,\left\{\mathcal{R}_{l}(P): P \subseteq \mathrm{P}, l \in\{1\}\right\}\right)$ such that, $\mathcal{R}_{1}(\emptyset)=U \times U$, for all $\emptyset \neq P \subseteq \mathrm{P}, \mathcal{R}_{1}(P)=\bigcap_{\mathrm{p} \in P} \mathcal{R}_{1}(\{\mathrm{p}\})$ and for all $\mathrm{p} \in \mathrm{P}, \mathcal{R}_{1}(\{\mathrm{p}\})$ is (resp. weakly) reflexive and symmetrical. Following the lines of the previous sections, it can be shown that truc is a minimal nice pair function with respect to $\mathrm{S}_{0}$ and $\mathcal{F}_{\mathrm{S}_{0}}$. Hence $\mathcal{F}_{\mathrm{S}_{0}}$ is $\left(=, \mathrm{S}_{0}\right)$-inf-representable in $\mathcal{I S}$. Moreover, let $S=(O B, A T) \in \mathcal{I} \mathcal{S}$. Two objects $O_{1}$ and $o_{2}$ are said to be in relation of right orthogonality (resp. left orthogonality) with respect to $A \subseteq A T$ (in short $o_{1} \operatorname{rorth}(A) o_{2}$-resp. $o_{1} \operatorname{lorth}(A) o_{2}$ ) iff for all $a \in A$, $a\left(o_{1}\right) \subseteq\left(V_{a l} \backslash a\left(o_{2}\right)\right)\left(\right.$ resp. $\left.\left(V_{a l} \backslash a\left(o_{1}\right)\right) \subseteq a\left(o_{2}\right)\right)$. With LS, $\operatorname{rorth}(A)=m_{(O B, A)}\left(F_{0}^{\prime}\right)$ $\left(\right.$ resp. $\left.\operatorname{lorth}(A)=m_{(O B, A)}\left(F_{0}^{\prime}\right)\right)$ with $F_{0}^{\prime}=\forall f f\left(x_{1}\right) \subseteq-f\left(x_{2}\right)\left(\right.$ resp. $F_{0}^{\prime}=\forall f-f\left(x_{1}\right) \subseteq$ $\left.f\left(x_{2}\right)\right)$. Using Lemma 4.5 and the above construction, $\left.\{\mathcal{K}\urcorner: \exists \mathcal{K} \in \mathcal{F}_{\mathrm{S}_{0}}\right\}$ is $\left(=,\left\langle F_{0}^{\prime}\right\rangle\right)$-infrepresentable in $\mathcal{I S}$.

\section{An example of non-representability}

Until now, only representability results have been shown. However non representability results are also very interesting in order to understand the relevance of the notion of 
representability we introduced. The example below provides some insight about classes of information systems closed under subsystems and classes of $\Sigma$-frames closed under subframes. Although Proposition 6.1 might appear quite natural, it has some unexpected consequences (see for instance Corollary 6.2).

Proposition 6.1. Let $\Sigma$ be a signature, $\mathrm{S}$ be a $\Sigma$-specification, $X \subseteq \mathcal{F}_{\Sigma}$ and $Y \subseteq \mathcal{I} \mathcal{S}$ closed under subsystems. If $X$ is $(=, \mathrm{S})$-inf-representable in $Y$ then $X$ is closed under subframes.

Corollary 6.2 . Let $\Sigma$ be a signature, $\mathrm{S}$ be a strong $\Sigma$-specification and $X$ be a set of $\Sigma$-frame such that for all $l \in\{1, \ldots, k\}$,

- $\mathcal{R}_{l}(\emptyset)=U \times U$, for all $\emptyset \neq P \subseteq \mathrm{P}, \mathcal{R}_{l}(P)=\bigcap_{\mathrm{p} \in P} \mathcal{R}_{l}(\{\mathrm{p}\})$

- for all $\mathrm{p} \in \mathrm{P}, \mathcal{R}_{l}(\{\mathrm{p}\})$ is serial (resp. atomic, weakly dense, discrete).

$X$ is not $(=, \mathrm{S})$-inf-representable in $\mathcal{I} \mathcal{S}$.

Some interesting relationships might exist between subframe logics (see [Wol93]) and the logics characterized by classes of frames informationally representable by frames derived from a class of information systems closed under subsystems. However it is not in the scope of this work.

\section{References}

[Bal97] Ph. Balbiani. Axiomatization of logics based on Kripke models with relative accessibility relations. In E. Orłowska, editor, Incomplete Information: Rough Set Analysis, pages 553-578. Physica Verlag, Heidelberg, 1997.

[Bun67] M. Bunge. Scientific research I. The search for system. Springer, Berlin, 1967.

[CDCG93] G. Cattaneo, M. L. Dalla Chiara, and R. Giuntini. Fuzzy-intuitionistic quantum logic. Studia Logica, 52:1-24, 1993.

[Che80] B. Chellas. Modal Logic. Cambridge University Press, 1980.

[CN89] G. Cattaneo and G. Nistico. Brouwer-Zadeh posets and three-valued Lukasiewicz posets. Fuzzy sets and Systems, 33:165-190, 1989.

[Gol91] R. Goldblatt. Semantic analysis of orthologic. In R. Goldblatt, editor, Mathematics of Modality, pages 81-97. CSLI Lecture Notes 43, Stanford, California, 1991.

[Gol92] R. Goldblatt. Logics of Time and Computation. Lecture Notes 7, CSLI Stanford, 2d edition, 1992.

[GT75] R. Goldblatt and S. Thomason. Axiomatic classes in propositional modal logic. In J. Crossley, editor, Algebra and Logic, pages 163-173. Springer-Verlag, Lecture Notes in Mathematics 450, 1975.

[HC84] G. Hughes and M. Cresswell. A companion to modal logic. Methuen, 1984.

[HM92] J. Halpern and Y. Moses. A guide to completeness and complexity for modal logics of knowledge and belief. Artificial Intelligence, 54:319-379, 1992.

[Kri63] S. Kripke. Semantical considerations on modal logics. Modal and Many-valued logics, Acta Philosophica Fennica, 1963.

[OP84] E. Orłowska and Z. Pawlak. Representation of nondeterministic information. Theoretical Computer Science, 29:27-39, 1984. 
[Orło85] E. Orłowska. Logic of nondeterministic information. Studia Logica, 44:93-102, 1985.

[Orło88a] E. Orłowska. Kripke models with relative accessibility and their applications to inferences from incomplete information. In G. Mirkowska and H. Rasiowa, editors, Mathematical Problems in Computation Theory, pages 329-339. Banach Center Publications, Volume 21 PWN - Polish Scientific Publishers, Warsaw, 1988.

[Orło88b] E. Orłowska. Logical aspects of learning concepts. Journal of Approximate Reasoning, 2:349-364, 1988.

[Orło89] E. Orłowska. Logic for reasoning about knowledge. Zeitschr. f. math. Logik und Grundlagen d. Math., 35:559-568, 1989.

[Orło93] E. Orłowska. Rough set semantics for non-classical logics. In W. Ziarko, editor, 2nd International Workshop on Rough Sets and Knowledge Discovery, Banff, Canada, pages 143-148, 1993.

[Orło94] E. Orłowska. Indiscernibility and orthogonality: two paradigms of incompleteness. In 3rd International Workshop on Rough Sets and Soft Computing, San Jose, California, pages $64-71,1994$.

[Orło95] E. Orłowska. Information algebras. In AMAST'95, Montreal, pages 50-65. LNCS 639, Springer-Verlag, 1995.

[Orło98] E. Orłowska (ed.). Logic at Work. Essays Dedicated to the Memory of Helena Rasiowa. Physica Verlag, Heidelberg, 1998. To appear.

[Paw83] Z. Pawlak. Information systems. Theoretical Foundations, 1983. WNT, Warsaw (in Polish).

[Paw91] Z. Pawlak. Rough Sets - Theoretical Aspects of Reasoning about Data. Kluwer Academic Press, 1991.

[Vak87] D. Vakarelov. Abstract characterization of some knowledge representation systems and the logic NIL of nondeterministic information. In Ph. Jorrand and V. Sgurev, editors, Artificial Intelligence: Methodology, Systems, Applications, pages 255-260. Elsevier Science Publishers B.V. (North-Holland), 1987.

[Vak89] D. Vakarelov. Modal logics for knowledge representation systems. In A. R. Meyer and M. Taitslin, editors, Symposium on Logic Foundations of Computer Science, PereslavlZalessky, pages 257-277. Springer-Verlag, LNCS 363, July 1989.

[Vak91a] D. Vakarelov. Logical analysis of positive and negative similarity relations in property systems. In M. de Glas and D. Gabbay, editors, First World Conference on the Fundamentals of Artificial Intelligence, 1991.

[Vak91b] D. Vakarelov. A modal logic for similarity relations in Pawlak knowledge representation systems. Fundamenta Informaticae, 15:61-79, 1991.

[Vak95] D. Vakarelov. A duality between Pawlak's information systems and bi-consequence systems with applications to first-order and modal characterizations of some informational relations. In M. de Glas and Z. Pawlak, editors, Second World Conference on the Fundamentals of Artificial Intelligence, pages 417-429. Angkor, July 1995.

[Vak97] D. Vakarelov. Information systems, similarity and modal logics. In E. Orłowska, editor, Incomplete Information: Rough Set Analysis, pages 492-550. Physica Verlag, Heidelberg, 1997.

[vB84] J. van Benthem. Correspondence Theory. In D. Gabbay and F. Günthner, editors, Handbook of Philosophical Logic, Volume II, pages 167-247. Reidel, Dordrecht, 1984.

[Wol93] F. Wolter. Lattices of modal logics. PhD thesis, University of Berlin, August 1993. 